\title{
Drug-coated balloon for the management of coronary chronic total occlusions
}

\author{
Erick Sanchez-Jimenez ${ }^{1}$, Rami El-Mokdad ${ }^{2}$, Rima Chaddad ${ }^{3}$, Bernardo Cortese ${ }^{1,4, *}$ \\ ${ }^{1}$ Cardiac department, Clinica Polispecialistica San Carlo, 20037 Milano, Italy \\ ${ }^{2}$ Cardiac department, Al-Zahraa University Hospital, 00961 Beirut, Lebanon \\ ${ }^{3}$ Cardiac department, Lebanese University, 00961 Beirut, Lebanon \\ ${ }^{4}$ Fondazione Ricerca e Innovazione Cardiovascolare, 20100 Milano, Italy \\ *Correspondence: bcortese@gmail.com (Bernardo Cortese) \\ Academic Editor: Alaide Chieffo \\ Submitted: 6 November 2021 Revised: 9 December 2021 Accepted: 17 December 2021 Published: 24 January 2022
}

\begin{abstract}
There is a higher rate of successful recanalization of patients with coronary chronic total occlusions, nevertheless, the rate of patients referred for revascularization remains low. In addition, there is a greater need to improve long-term outcomes of chronic total occlusions after percutaneous coronary intervention, and although the implantation of new-generation drug-eluting stents has been optimized with coronary imaging guidance, the rate of stenting failure remains a major issue and long-term vessel patency could be improved. We reviewed clinical data regarding the benefit of treating chronic total occlusions and the use of drug-coated balloons as an alternative therapeutic modality in this setting. Although clinical data is limited, the initial evidence and the daily clinical practice point towards a synergistic hybrid treatment strategy based on the combination of drug-coated balloons plus drug-eluting stents, reducing the total stent length and maintaining the scaffolding properties of stents where it is mandatory. Additionally, drug-coated balloons allow natural enlargement of the open vessel after percutaneous coronary intervention (PCI), which is a major limitation of stents in chronic total occlusion (CTO).
\end{abstract}

Keywords: Drug-coated balloon; Chronic total occlusion; Coronary artery disease; Percutaneous coronary intervention

\section{Introduction}

Interventional cardiology is evolving rapidly with an increased need to improve the outcomes of the treatment of coronary artery disease which is the leading cause of death worldwide. Native coronary chronic total occlusions (CTO) are identified in $18 \%$ to $20 \%$ of coronary angiograms with significant disease and with an incidence that increases with age $[1,2]$. However less than half of such patients will undergo revascularization, either by coronary artery bypass graft (CABG) or by percutaneous coronary intervention (PCI), as most patients are being treated medically [3]. This cautious approach is possibly associated with the fear of periprocedural complications, the challenging skills required to recanalize a totally occluded artery and some doubts about the long-term clinical outcome after long stenting. PCI with stenting in the modern era has been the mainstay management for CTO; however, the longer the stents are implanted, the greater the risk of late adverse events [4].

Drug-coated balloons (DCB) have been used as an alternative to the drug-eluting stent (DES) in this setting, but yet evidence-based medicine is still scarce on the matter $[5,6]$.

\section{PCI for CTO-an overview}

Coronary CTO can be defined as interruption of antegrade coronary flow with Thrombolysis In Myocardial Infarction (TIMI) grade 0 and a stenosis of $100 \%$ older than three months [3]. Right coronary artery is the more commonly affected vessel. The incidence is higher for patients with history of CABG compared to naïve patients [2].

Recanalization of CTO is technically challenging and PCI carries a higher risk of complications than for nonCTO lesions. New techniques have been developed to cross the lesion in an anterograde, retrograde and hybrid fashion, with better success rates. The recanalization rate has been improved to $85-90 \%$ in most experience centers using new algorithms with guidewire escalation, including the use of intravascular ultrasound (IVUS). Additionally, the safety of this procedure has also recently improved, with intraprocedure complications occurring in less than $3 \%$ of cases $[7,8]$.

Until today, it is normal to perform CTO-PCI implanting only new-generation DES, due to the good clinical results shown by this technology and the high radial force warranted in the immediate. The use of intravascular imaging, especially IVUS and rarely optical coherence tomography (OCT), is also increasing in order to optimize device implantation. Despite all these improvements have ensured greater immediate performance and minimized the risk of short-term complications related to the stent implantation 
process, the stent failure rate remains as high as $15-20 \%$ in the long term, due to a higher rate of stent thrombosis and a higher need for revascularization $[4,9,10]$. In highly calcified lesions, the risk of stent underexpansion is quite common, despite the increasing use of atherectomy and intravascular lithotripsy [4].

Moreover, the management of CTO with long stenting generally requires to place the patient on long-term prophylactic dual antiplatelet therapy (DAPT), which is associated with an increased risk of complications, such as bleeding and mortality, especially in frail and elderly patients. The risk of major bleeding is doubled with DAPT compared to single antiplatelet therapy, including the risk of intracranial hemorrhage, which is the most serious complication [11].

\section{Is there any benefit of PCI in a CTO?}

Symptoms and improved quality of life remain the main indication for CTO-PCI, which are strongly supported by observational and randomized controlled trials [12].

In a multicenter randomized study with the aim to compare coronary revascularization with optimal medical therapy (OMT) for the treatment of CTO (EuroCTO), 396 patients were randomly assigned to CTO-PCI vs. OMT alone. Greater improvement was observed in the subscales of Seattle angina questionnaire at 12 months with PCI compared with OMT for angina frequency $(5.23, p=0.003)$, and quality of life $(6.62, p=0.007)$ [13].

In a single-center randomized study, IMPACTORCTO, 94 patients with totally occluded right coronary artery (isolated) were randomly assigned to CTO-PCI vs. OMT. Myocardial ischemic burden decreased significantly from $27.7 \pm 8.5 \%$ at baseline to $16.1 \pm 8.6 \%$ at 12 months ( $p$ $<0.01)$ in the PCI group, whereas no significant improvement were recorded in the OMT group (from $28.4 \pm 8.6 \%$ at baseline to $27.0 \pm 8.0 \% ; p=0.83$ ). Moreover, sixminute walking distance was significantly increased in the PCI group [14].

One trial (REVASC) aimed to demonstrate improvement in left ventricular function after CTO revascularization and showed a reduction in major adverse cardiac events (MACE) at 12 moths, mainly driven by the reduction of repeated interventions in the PCI group compared to the OMT group $(16.3 \%$ vs. $5.9 \% ; p=0.02)$, although the primary endpoint was not achieved [15].

Another recent trial (DECISION-CTO) with a primary endpoint accounting for combined mortality, myocardial infarction, stroke and any revascularization did not reveal any statistical difference between group events at 4 years follow-up $(22.3 \%$ in the PCI group vs. $22.4 \%$ in the OMT group, $p=0.86$ ) probably due to low power and high crossover rates [16].

In a large-scale observational study in 592 patients with treated CTO, Gong et al. [17] showed a statistically significant improvement in cardiac death and MACE after successful revascularization with a hazard ratio of 0.239 for cardiac death and 0.541 for MACE

A meta-analysis that included 25 studies comparing successful CTO-PCI versus failed PCI in 28,486 patients with a median follow-up of 3.11 years, demonstrated lower mortality, less residual angina, lower risk of stroke, and less need of subsequent CABG in case of success (odds ratio of $0.52,0.38,0.72$ and 0.18 , respectively) [18].

Two registries also showed an improvement in mortality. In the Italian CTO Registry Tomasello et al. [19] examined the long-term outcomes of 1777 CTO patients treated with PCI, OMT and surgery. Patients undergoing PCI showed lower rates of MACE and cardiac death at 1 year of follow-up compared to patients treated with OMT or undergoing CABG; MACE was $2.6 \%$ vs. $8.2 \%$ vs. $6.9 \%$, respectively; $p<0.001$ and $p<0.01$ vs. PCI, and cardiac death was $1.4 \%$ vs. $4.7 \%$ vs. $6.3 \%$; $p<0.001$ and $p<$ 0.001 vs. PCI. After propensity score-matching analysis, PCI showed a lower incidence of cardiac death (1.5\% vs. $4.4 \%, p<0.001)$, myocardial infarction (1.1\% vs. $2.9 \%, p$ $=0.03)$ and hospitalization $(2.3 \%$ vs. $4.4 \%, p=0.04)$ compared to OMT.

In the Swedish Coronary Angiography and Angioplasty Registry (SCAAR), the existence of CTO in a patient was correlated as an independent mortality factor with a hazard ratio of $1.29, p<0.001$ and patients with an effective recanalization attempt were associated with significantly improved survival with a hazard ratio of $0.85, p<$ 0.034 [20].

\section{Drug coated balloons-technical background}

In this context, DCB may offer an attractive therapeutic modality since these devices allow for local drug delivery of the antiproliferative agents directly into the coronary artery wall after single balloon inflation, with the benefit of not leaving metal implants in place and avoid the potential long-term complications encountered with DES (Fig. 1) $[21,22]$.

DCB was developed to avoid insertion of a permanent foreign body into the coronary vessel, and thus prevent any potential problem caused by stents. DCB is a semicompliant balloon coated with an anti-proliferative drug, which is rapidly released through an excipient when inflated [23,24]. Most CE-marked manufactured DCB use paclitaxel, due to its affinity to lipid tissue and on-site retention features.

Paclitaxel is a natural diterpenoid found in Taxus species. It binds to the microtubule subunit of the cell during mitosis and has local, potent, and dose-dependent inhibitory effects on arterial smooth muscle cells, preventing proliferation and migration, hence battling neointimal hyperplasia. Most DCBs are coated with $3 \mathrm{microg} / \mathrm{mm}^{2}$, which is the optimal efficacy dose as shown in animal models [23,24]. Sirolimus-coated balloons represent a novel technology introduced in 2016 in the field of interventional 


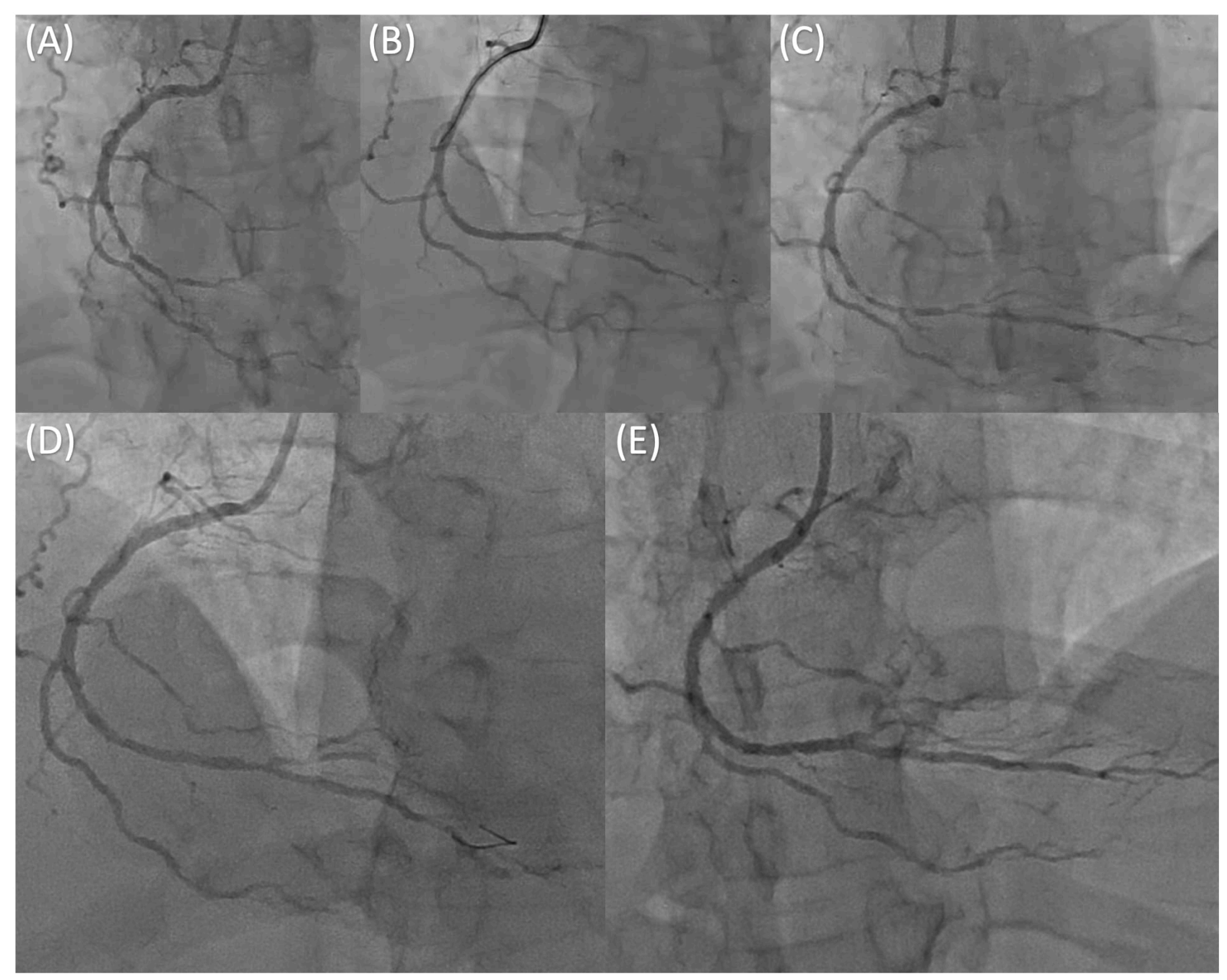

Fig. 1. High bleeding risk patient with right coronary artery chronic total occlusion treated with DCB-only (first generation) strategy. (A) Left oblique view of RCA CTO. (B) Final angiographic result after recanalization, lesion preparation and PCI with paclitaxel DCB $2.0 \mathrm{~mm}$. (C) 5-months later the patient presented with recurrent angina and the coronary angiography showed DCB failure. (D) Final angiographic result after PCI with new generation DCB. (E) After 6-months coronary angiography showed vessel healing with significant lumen gain.

cardiology; sirolimus reversibly binds to the FKBP 12 protein and potently inhibits cell proliferation by organizing a complex with the mammalian target of rapamycin, thus obstructing cell cycle function between the G1 and S phases of mitosis. This drug has more sustained antiproliferative effect during hypoxia and less risk for systemic toxic effects compared to paclitaxel [25]. Currently, there are 3 sirolimus DCBs marketed in Europe [26,27]. Furthermore, clinical data on sirolimus DCB is still scarce compared to paclitaxel DCB.

PCI with DCB should be perform only after adequate CTO opening and lesion preparation with all the necessary tools to achieve an optimal result, which helps to determine the real size of the vessel and improve deliver capacity. Prior to DCB delivery, the vessel must be assessed to achieve $<30 \%$ residual stenosis, TIMI flow grade 3 and non-flow limiting dissections. The DCB balloon can be $1: 1$ or $0.8: 1$ size of the native vessel; low pressure inflation and prolong time is recommended. After DCB delivery, the vessel must be evaluated with the same characteristics explained above regarding lesion preparation. In these are not met, bailout stenting is recommended $[23,28]$.

\section{Clinical stand point of DCB use for CTO intervention}

Data on DCB in CTO is still scarce with no randomized trials available comparing directly DCB versus DES, but one trial showed the feasibility of DCB plus bare-metal stent against paclitaxel-eluting stent [29-31]. On top of the feasibility of this strategy as depicted by several case series, 2 cohort studies were published to date. The first was a multicenter cohort study of 34 patients with one native 


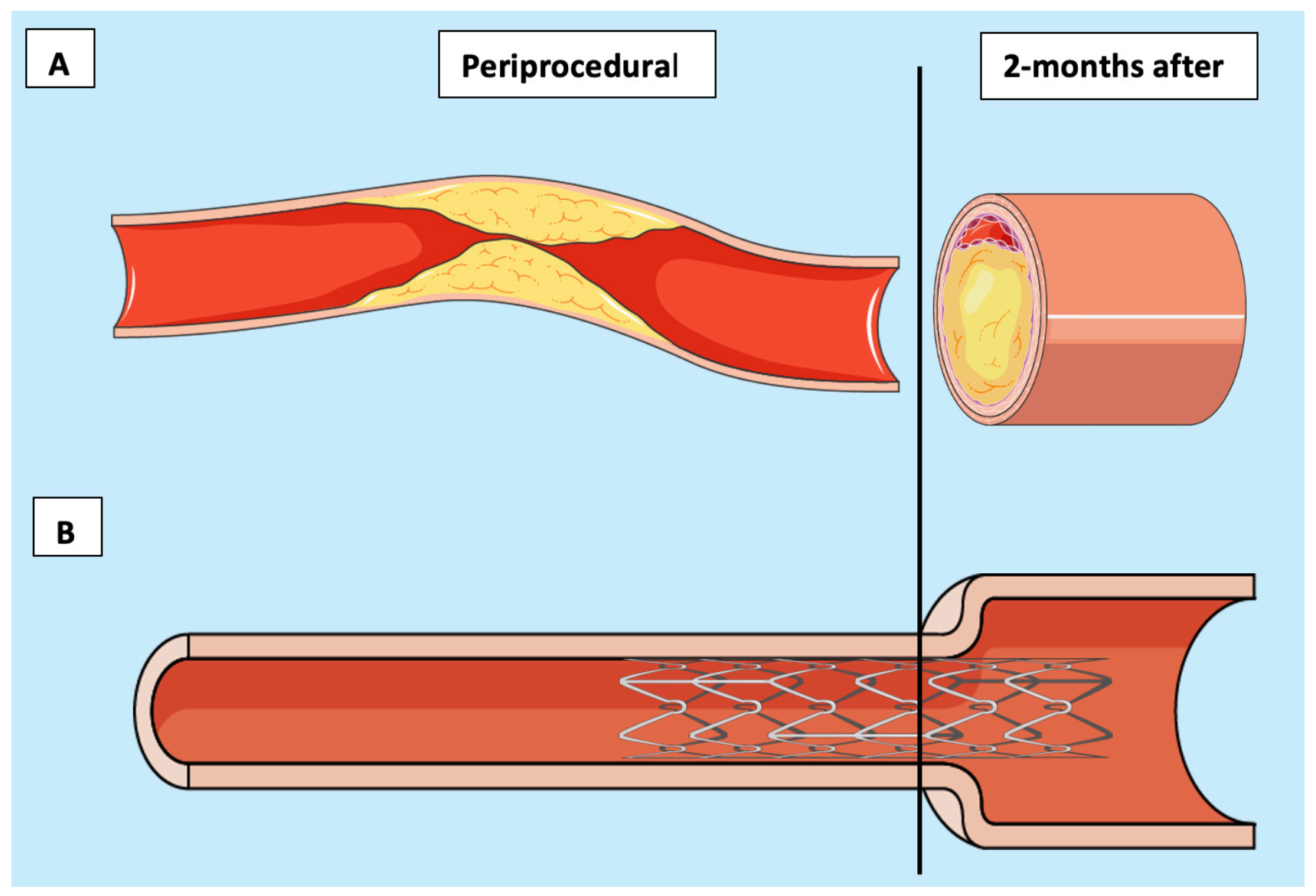

Fig. 2. Comparison between CTO-PCI results overtime. (A) Investment procedure: on the left, a coronary artery with chronic total occlusion failed to recanalized after first attempt with multiple wires. Then was decided to perform scratch-and-go technique and drugcoated balloon inflation. On the right the same coronary artery 2 months later showing reduced tissue proliferation and maintaining anterograde flow, improving the chances for a second attempt. (B) CTO-PCI and late malapposition: the left portion of the stent with good apposition to the vessel wall after a successful CTO-PCI. 2 months later the right portion of the stent showing the real vessel diameter and positive remodeling, leaving some gap between the stent and the vessel wall, which increases the chance to stent thrombosis and instent restenosis.

CTO recanalized and treated with only-DCB (SeQuent Please, B. Braun, Germany) strategy, recanalization was considered satisfactory only in $79.4 \%$ (27) of patients. Restenosis and reocclusion occurred in 11.8\% (4) and 5.9\% (2), respectively, of the total of 34 patients treated. Only 1 patient had restenosis and 1 reocclusion. A luminal enlargement was found in $67.6 \%$ of the patients with a mean late luminal gain of $0.11 \pm 0.49 \mathrm{~mm}$. A clinical improvement was observed with significant angina class reduction from baseline $(p<0.001)$. No death or myocardial infarction was recorded [5].

The second was a single-center cohort study conducted in United Kingdom, which included 41 patients and 44 CTO lesions with a Japanese-CTO score of $\geq 2$ in $34.1 \%$; all patients were treated with only DCB strategy (SeQuent Please). De novo lesions represented only $8 \%$ and $43.2 \%$ were multi-vessel disease. The anterograde wiring technique was successful in $97.7 \%$. The mean DCB diameter used was $2.8 \pm 0.6 \mathrm{~mm}$ and the mean length of treated coro- nary segment was $41 \pm 26 \mathrm{~mm}$. Fifteen (34.1\%) lesions resulted in coronary dissections, however only $2(4.5 \%)$ of them required bailout stenting. The follow-up period was approximately 1 year. Authors reported 1 case of noncardiac death, 3 cases $(6.8 \%)$ of target lesion revascularization, and $1(2.3 \%)$ target vessel revascularization. The overall MACE rate was 9.8\% [6].

Finally, a recent interesting report of 3 cases with more complex CTO lesions in native vessel disease showed the safety and feasibility of DCB in this scenario. Investigators used DCB during the procedure after failure of recanalization in the first attempt, reducing tissue proliferation, maintaining anterograde flow and improving healing of the dissection flaps (Fig. 2A) [20].

The potential role of DCB to act as adjuvant or definite treatment for CTO is promising. Large proportion of subjects presenting with CTO are suffering multivessel coronary artery disease, the majority associated with significant comorbidities and a high bleeding risk. Limiting the total 


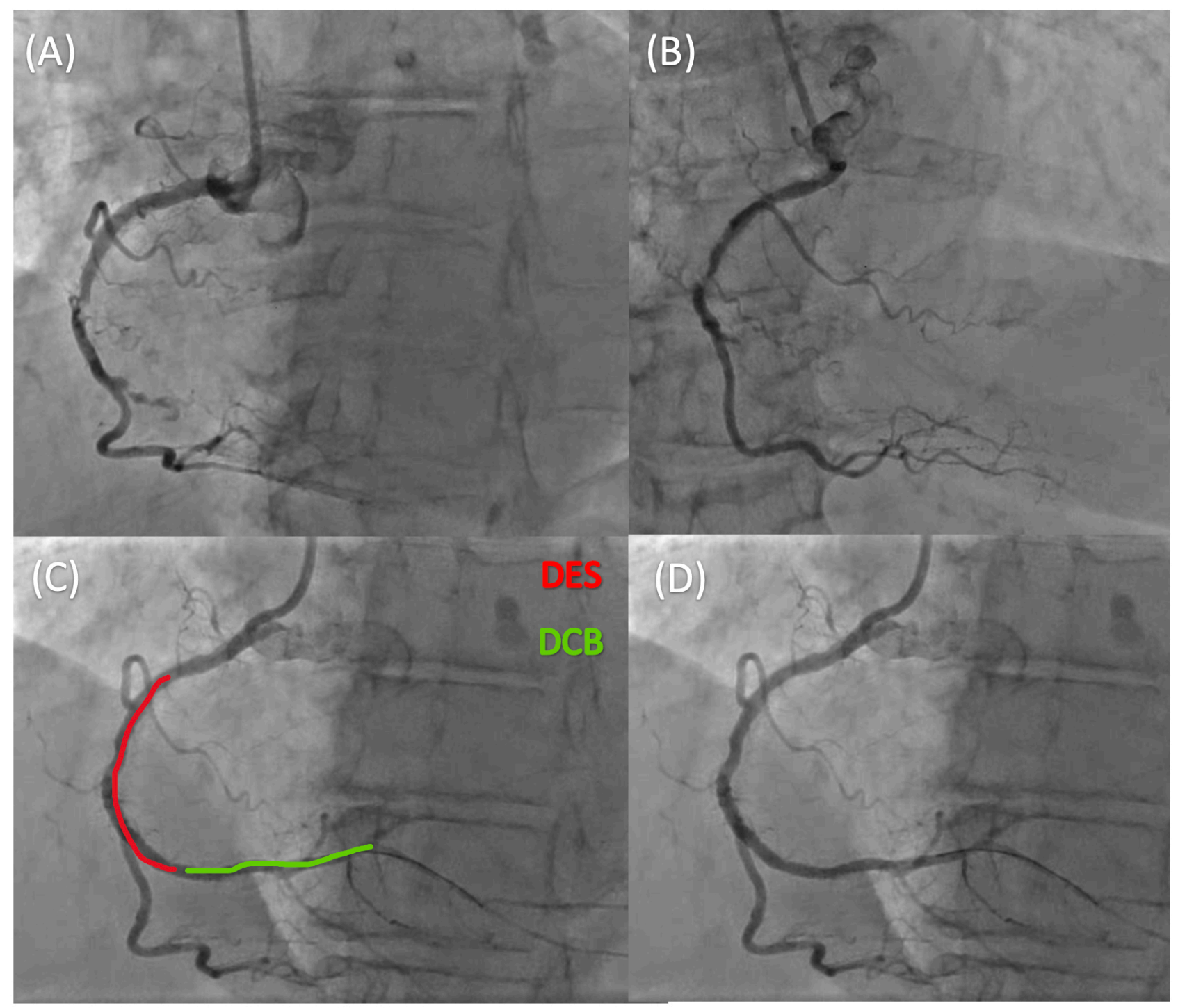

Fig. 3. Hybrid approach with DES and DCB. (A) Right coronary artery chronic total occlusion. (B) Cranial view showing the proximal cap. (C) Drug-eluting stent implantation for the mid segment and drug-coated balloon for the distal segment. (D) Angiographic final result.

stent length in complex higher-risk and indicated patients with native coronary disease seems to be associated with better clinical outcomes [21].

Another potential advantage is the theoretical lower risk of late or very late stent malapposition and subsequent adverse events, considering the frequent misunderstanding of correct vessel size during CTO recanalization and the subsequent not rare failure in choosing the correct stent sizing. Moreover, it is hypothetically easier to recanalize a vessel in case of re-occlusion after DCB instead of DES. It should also be acknowledged that some DCB (all eluting paclitaxel, till date) have shown an interesting action in terms of late lumen enlargement due to a direct effect on the tunica adventitia, a very interesting milieu of this technology for the treatment of CTO (Fig. 2B) [22].
Furthermore, as mention above, intravascular imaging especially IVUS can act as adjuvant to recanalization of CTOs, but also (IVUS and OCT) as a tool to determine if the small caliber of the distal epicardial coronary is due to hypoperfusion versus dissection versus atherosclerosis and find the real vessel diameter, lesion length and plaque characteristics in order to select the best DCB or stent [7].

\section{Conclusions}

Although clinical data supporting the use of DCB in CTO lesions is limited, initial evidence, daily clinical practice and solid concepts point towards a synergistic hybrid treatment strategy based on the combination of DCB+DES, reducing the total stent length and still maintaining the scaffolding properties of DES where it is mandatory, especially in complex patients (Fig. 3). One clinical trial (clinical- 
trails.gov/NCT04881812) will evaluate the use of DCB for the distal portion of the CTO with spot stenting of the proximal segment of the lesion. Finally, DCB-only approach in CTO manage with anterograde lumen-to-lumen wiring is feasible but larger studies are needed and the performance of DCBs in this setting will be tested in the multicenter randomized PICCOLETO III trial, comparing DES to paclitaxel and sirolimus DCB.

\section{Author contributions}

ES-J-clinical stand point of DCB use for CTO intervention Fig. 2, case for Fig. 3, review; RE-Mabstract, introduction, PCI for CTO-an overview; RCDCB-technical background, benefit of PCI in CTO; BCcase for Fig. 1, conclusion, final review. All authors contributed to editorial changes in the manuscript. All authors read and approved the final manuscript.

\section{Ethics approval and consent to participate}

Not applicable.

\section{Acknowledgment}

Thanks to all the peer reviewers for their opinions and suggestions.

\section{Funding}

This research received no external funding.

\section{Conflict of interest}

The authors declare no conflict of interest. Bernardo Cortese is serving as one of the Guest editors of this journal. We declare that Bernardo Cortese had no involvement in the peer review of this article and has no access to information regarding its peer review. Full responsibility for the editorial process for this article was delegated to Alaide Chieffo.

\section{References}

[1] Fefer P, Knudtson ML, Cheema AN, Galbraith PD, Osherov AB, Yalonetsky S, et al. Current Perspectives on Coronary Chronic Total Occlusions. Journal of the American College of Cardiology. 2012; 59: 991-997.

[2] Azzalini L, Jolicoeur EM, Pighi M, Millán X, Picard F, Tadros V, et al. Epidemiology, Management Strategies, and Outcomes of Patients with Chronic Total Coronary Occlusion. The American Journal of Cardiology. 2016; 118: 1128-1135.

[3] Galassi AR, Werner GS, Boukhris M, Azzalini L, Mashayekhi $\mathrm{K}$, Carlino $\mathrm{M}$, et al. Percutaneous recanalisation of chronic total occlusions: 2019 consensus document from the EuroCTO Club. EuroIntervention. 2019; 15: 198-208.

[4] Mahadevan K, Cosgrove C, Strange JW. Factors Influencing Stent Failure in Chronic Total Occlusion Coronary Intervention. Interventional Cardiology: Reviews, Research, Resources. 2021; 16: e27.

[5] Köln PJ, Scheller B, Liew HB, Rissanen TT, Ahmad WAW, Weser R, et al. Treatment of chronic total occlusions in native coronary arteries by drug-coated balloons without stenting - a feasibility and safety study. International Journal of Cardiology. 2016; 225: 262-267.

[6] Wickramarachchi U, Corballis NH, Maart CA, Gilbert TJ, Eccleshall SC. 24 Drug coated balloon-only angioplasty in chronic total occlusions, a UK single centre experience. Heart. 2017; 103: A11.

[7] Xenogiannis I, Tajti P, Karmpaliotis D, Garbo R, Gagnor A, Burke MN, et al. Intravascular Imaging for Chronic Total Occlusion Intervention. Current Cardiovascular Imaging Reports. 2018; 11: 31 .

[8] Wu EB, Brilakis ES, Mashayekhi K, Tsuchikane E, Alaswad K, Araya M, et al. Global Chronic Total Occlusion Crossing Algorithm. Journal of the American College of Cardiology. 2021; 78: 840-853.

[9] Mehran R, Claessen BE, Godino C, Dangas GD, Obunai K, Kanwal $\mathrm{S}$, et al. Long-term outcome of percutaneous coronary intervention for chronic total occlusions. JACC: Cardiovascular Interventions. 2011; 4: 952-961.

[10] Walsh SJ, Hanratty CG, McEntegart M, Strange JW, Rigger J, Henriksen PA, et al. Intravascular Healing is not Affected by Approaches in Contemporary CTO PCI. JACC: Cardiovascular Interventions. 2020; 13: 1448-1457.

[11] Ha ACT, Bhatt DL, Rutka JT, Johnston SC, Mazer CD, Verma S. Intracranial Hemorrhage during Dual Antiplatelet Therapy. Journal of the American College of Cardiology. 2021; 78: 13721384.

[12] Brilakis ES, Mashayekhi K, Tsuchikane E, Abi Rafeh N, Alaswad K, Araya M, et al. Guiding Principles for Chronic Total Occlusion Percutaneous Coronary Intervention. Circulation. 2019; 140: 420-433.

[13] Werner GS, Martin-Yuste V, Hildick-Smith D, Boudou N, Sianos $\mathrm{G}$, Gelev V, et al. A randomized multicentre trial to compare revascularization with optimal medical therapy for the treatment of chronic total coronary occlusions. European Heart Journal. 2018; 39: 2484-2493.

[14] Obedinskiy AA, Kretov EI, Boukhris M, Kurbatov VP, Osiev AG, Ibn Elhadj Z, et al. The IMPACTOR-CTO Trial. JACC: Cardiovascular Interventions. 2018; 11: 1309-1311.

[15] Mashayekhi K, Nührenberg TG, Toma A, Gick M, Ferenc M, Hochholzer W, et al. A Randomized Trial to Assess Regional Left Ventricular Function after Stent Implantation in Chronic Total Occlusion. JACC: Cardiovascular Interventions. 2018; 11: 1982-1991.

[16] Lee S, Lee PH, Ahn J, Park D, Yun S, Han S, et al. Randomized Trial Evaluating Percutaneous Coronary Intervention for the Treatment of Chronic Total Occlusion. Circulation. 2019; 139: 1674-1683.

[17] Gong X, Zhou L, Ding X, Chen H, Li H. The impact of successful chronic total occlusion percutaneous coronary intervention on long-term clinical outcomes in real world. BMC Cardiovascular Disorders. 2021; 21: 182.

[18] Christakopoulos GE, Christopoulos G, Carlino M, Jeroudi OM, Roesle M, Rangan BV, et al. Meta-Analysis of Clinical Outcomes of Patients who Underwent Percutaneous Coronary Interventions for Chronic Total Occlusions. The American Journal of Cardiology. 2015; 115: 1367-1375.

[19] Tomasello SD, Boukhris M, Giubilato S, Marzà F, Garbo R, Contegiacomo G, et al. Management strategies in patients affected by chronic total occlusions: results from the Italian Registry of Chronic Total Occlusions. European Heart Journal. 2015; 36: 3189-3198.

[20] Råmunddal T, Hoebers LP, Hoebers L, Henriques JPS, Dworeck $\mathrm{C}$, Angerås $\mathrm{O}$, et al. Chronic total occlusions in Sweden-a report from the Swedish Coronary Angiography and Angioplasty Registry (SCAAR). PLoS ONE. 2014; 9: e103850.

[21] Cortese B, Testa L, Di Palma G, Heang TM, Bossi I, Nuruddin $\mathrm{AA}$, et al. Clinical performance of a novel sirolimus-coated bal- 
loon in coronary artery disease: EASTBOURNE registry. Journal of Cardiovascular Medicine. 2021; 22: 94-100.

[22] Cortese B, Di Palma G, Guimaraes MG, Piraino D, Orrego PS, Buccheri D, et al. Drug-Coated Balloon Versus Drug-Eluting Stent for Small Coronary Vessel Disease. JACC: Cardiovascular Interventions. 2020; 13: 2840-2849.

[23] Cortese B, Berti S, Biondi-Zoccai G, Colombo A, Limbruno U, Bedogni F, et al. Drug-coated balloon treatment of coronary artery disease: a position paper of the Italian Society of Interventional Cardiology. Catheterization and Cardiovascular Interventions. 2014; 83: 427-435.

[24] Alfonso F, Scheller B. State of the art: balloon catheter technologies - drug-coated balloon. EuroIntervention. 2017; 13: 680 695.

[25] Chen Y, Zeng Y, Zhu X, Miao L, Liang X, Duan J, et al. Significant difference between sirolimus and paclitaxel nanoparticles in anti-proliferation effect in normoxia and hypoxia: the basis of better selection of atherosclerosis treatment. Bioactive Materials. 2021; 6: 880-889.

[26] Jeger RV, Eccleshall S, Wan Ahmad WA, Ge J, Poerner TC, Shin $\mathrm{E}$, et al. Drug-Coated Balloons for Coronary Artery Disease. JACC: Cardiovascular Interventions. 2020; 13: 1391-1402.
[27] Cortese B, Caiazzo G, Di Palma G, De Rosa S. Comparison between Sirolimus- and Paclitaxel-Coated Balloon for Revascularization of Coronary Arteries: the SIRPAC (SIRolimusPAClitaxel) Study. Cardiovascular Revascularization Medicine. 2021; 28: 1-6.

[28] Cortese B, Sanchez-Jimenez E. Back to the future: DCB use instead of DES for the treatment of complex, native coronary artery disease. European Heart Journal Supplements. 2021; 23: E63-E67.

[29] Wöhrle J, Werner GS. Paclitaxel-coated balloon with bare-metal stenting in patients with chronic total occlusions in native coronary arteries. Catheterization and Cardiovascular Interventions. 2013; 81: 793-799.

[30] Ybarra LF, Dandona S, Daneault B, Rinfret S. Drug-coated balloon after subintimal plaque modification in failed coronary chronic total occlusion percutaneous coronary intervention: a novel concept. Catheterization and Cardiovascular Interventions. 2020; 96: 609-613.

[31] Cortese B, Silva Orrego P, Agostoni P, Buccheri D, Piraino D, Andolina G, et al. Effect of Drug-Coated Balloons in Native Coronary Artery Disease Left with a Dissection. JACC: Cardiovascular Interventions. 2015; 8: 2003-2009. 